\title{
A Path Based Model for Sonification
}

\author{
Keith M. Franklin, Jonathan C. Roberts \\ Computing Laboratory, University of Kent at Canterbury, England \\ $\{$ kmf1@kent.ac.uk,j.c.roberts@kent.ac.uk\}
}

\begin{abstract}
Recently researchers have been interested in nonvisual forms of presentation. Sound, touch, smell, as well as vision can be used to depict information. One important medium is sound; it can encode information for the blind or partial sighted, used to display information when it is impossible to use a screen, and the hardware is cheap and widely available. Researchers have investigated sonifying various data sources with different data types and configurations. In this paper we present a novel path-based model that can be used to describe each of these different sonifications. In summary, the path dictates the sonification tour, the data is mapped into sound via a transfer function, and the quantity of information being sonified is determined by both the span and how the abstract path is registered to the data.
\end{abstract}

Keywords--- Sonification, sonifying images, path based sonification, non-visual representations.

\section{Introduction}

In recent years, there has been a growing interest into non-visual techniques such as sound, touch and smell to represent information. For example, line graphs of stock market data can be represented by sound (the day to day growth by time, and the stock value represented by a pitch change) and the same information could be represented by a tactile display (allowing the user to feel the increase or decline of the stock).

Accessibility is one of the main motivations to this work, such that the presented information can be understood by those with disabilities such as bline or partially sighted. However these non-visual forms are useful for other situations. For instance, haptic representations are useful for training (e.g. the fine kinesthetic movements surgeons need to learn) or remote vehicle operation (such may be useful by a bomb disposal team). Sonification can enhance the display of information on small screens, as used in phones or personal organizers, and importantly sonification is useful to display quickly changing data (as the ear is good at noticing subtle changes).
One important non-visual presentation medium is sonification. Sound is available on a wide variety of systems, therefore it is cheap to both develop sonification systems and for a user to use. However, much of the current sonification research has been unstructured (indeed this is a case facing all researchers of non-visual 'visualization'). Researchers have imagined new realizations, implemented these ideas and tested their systems, but have not sought to generalize and unify principles from related work. Thus in this work we propose a unifying model that unites and generalizes the current sonification approaches.

One of the most challenging aspects of creating new sonifications is to develop an appropriate mapping that will map an $n$-dimensional data set into sound (which is primarily a one-dimensional medium). For example, one challenge is to generate a sonifcation of an image. On strategy researchers have adopted is to sweep a line across the image, converting the pixels (that fall under the sweeping line) into sound. Other strategies have been developed for vector and object-based images (see the related work section 2). This has often caused researchers to create complex mappings; some are more understandable than others. The difference in usability may be due to the encoding style that the developers have adopted, with mappings that are too abstract for the listener to understand, merely inappropriate for the task in hand, or overload the user with too much irrelevant information.

We purpose a model that unifies all current methods and approaches to sonifying information. This model determines the sequential order, size of area that is sampled for sonification.

Both researchers and users will benefit from this model. Researchers will be able to utilize a standard model in which they can describe their sonifications, develop generic sonification systems, and explore new representation strategies. Using the model users will be able to select the sonification method that best presents the information, and comprehend the construction of the sonification and thus better understand the underlying information. In the future we foresee that automated systems can select the most appropriate sonification style based on an analysis of the input data. 
In this paper we (1) discuss the model in detail, (2) describe examples of how the model relates to current methods and how it can be used to create new approaches, and (3) briefly present and evaluate a prototype system based on the model.

\section{Background and related research}

Sonification has been applied to numerous application domains and datasets, including statistical information such as stock market data [1], network diagrams (such as central heating schematics) [2], algorithm presentation (e.g. sorting algorithms [3]) and physical data (such as oil and gas well sonification [4]).

In each of these representations, there are two independent stages that occur. First, a transfer function is generated that details how the data values are exchanged to sound values or the relationship between the sounds [5], this is equivalent to generating a color map for traditional graphics visualizations. Second, the developer needs to consider the order in which the data is to be played (in some situations, such as when displaying time-series data, this order is implicit). This latter stage, which is the focus of this paper, can be further subdivided into two general areas (1) query based methods, where the user continuously selects the information to be sonified, and (2) tours, where the information is arranged into a predefined order for sonification.

There are numerous transfer functions that could be applied. For instance higher values to higher pitches, $\mathrm{x}, \mathrm{y}$ space to localized sound position, categories to different timbres. These are abstract mappings, where any particular association must be learnt. Furthermore, auralization could be used instead of sonification, where the data value is mapped into the spoken word. An example of this would be the work done by Frankie James [6], who presented a method for auralizing both the contents and structure of HTML pages based on the concept of radio presenters (different content read by different voices). Although auralization has the advantage that users are not required to learn the mapping, it tends to be longer in duration, harder to realize complex information and can be annoying when used for prolonged periods of time. Therefore within this paper we concentrate on sonification and the use of abstract mappings.

\subsection{Query base methods}

Query based methods use a selector (such as a pointer) that is controlled by the user. When an area of information is selected the system generates an appropriate sound. An example of this approach is in the use of earcons [7], such as used by Helle et al [8] to represent mobile phone menus. As the user moves down the menu items so a unique motif is played. The earcons are structured musical motifs that are uniquely identifiable, in the case of the mobile phone menu; the motifs are carefully crafted such that users can perceive the location within the menu and sub-menus (e.g. each menu item exhibits similar traits to its parent).

Another example is the work by Bennett [2] who presented sonification of heating schematics. Two scales sonified the position of the nodes within the diagram, each in a different instrument and one for each axis, the number of notes in the scale corresponded to the position in along an axis. A final example of a query based approach is AudioGraf [9]. In this application users explore a graph by using a touch panel. As the user moves their finger around the touch pad, auditory information is presented based on the currently selected nodes or edges.

\subsection{Tour based methods}

The second method of encoding information is based on the concept of a tour. A tour arranges the data into a predefined order for sonification. For example, to represent a bar chart [10] the order of the categories (the bars themselves) is chosen, which is typically left to right along the $\mathrm{x}$-axis, and then a transfer function is applied to the ordered information, which maps the y-values to (say) pitch. In pie chart sonification [11], the tour describes the order in which the segments are sounded. This representation uses a transfer function that maps the start and end location of each segment to an equivalent location in the azimuth plane, which surrounds the user's head. Similarly, other mathematical visualizations have been sonified, such as line graphs [12].

Other more challenging sonifications include the representation of images. To sonify an image, the developer needs to work out an appropriate tour, which will convey all necessary information to the user.

For a vector image, the developer only needs to find a path that visits the individual components that are of interest. For instance if the image contained a series of geometric shapes, the tour might order the shapes left to right, top to bottom (sounding them out similarly to Bennett's central heating sonification [2]).

For a bitmap image (such as a digital camera image) the developer could either (1) provide a sonification tool that located and visited the main features of the image, sounding out the positions and values of various attributes, much like the eye moves saccadically from one feature to another; or (2) consecutively visit every pixel in the image applying the desired transfer function. This latter method has been used in a number of systems, e.g the vOICe system, as detailed by Jones [13]. Moreover, Jones mentions head-mounted cameras that exchange moving pictures from a video camera into sound.

\section{The model}

To help both researchers and users understand and select the most appropriate tour based methods, we have defined four terms path, span, span angle and span envelope. These terms form the bases of our model. The path expresses the ordering of the information, whilst the 
other three allow the developer to richly explain the how the information will be sonified. We believe this model can be used to express all current techniques in such a way that other users will be able to understand the relationship between the sonification and the underlying data, with the exception of the transfer function, which is independent to the tour.

\subsection{Path}

A path describes the order in which information will be presented to the listener. Within our model, a path is created from a series of path points. During the development of a new path each path point represents an arbitrary location, which is defined relative to other path points. When the path is applied to a data set, each path point maps to a tangible part of the dataset. This allows the path to be rescaled independently of the data. In principle the path can be placed in two or threedimensional space, however for descriptions within this paper we detail the paths in two-dimensions.

In addition, and unlike current techniques which consecutively order the information, each path point can be placed anywhere within the abstract space in a completely random order. For example, users could create paths that can compare different areas of their data, such as moving from one feature of interest to another in a bitmap image, alternatively the path may be used to describe a route from one geometric primitive to another for vector images.

Two different paths are shown in Figure 1. Figure 1(a) is an example of the path that would be used to create the sweeping line (also known as an occluding front), which is currently the most popular tour based method. Figure 1(b), shows a path that can provide an overview of the information over a wider area.

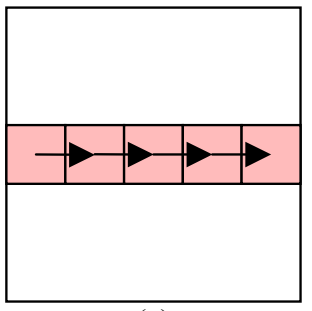

(a)

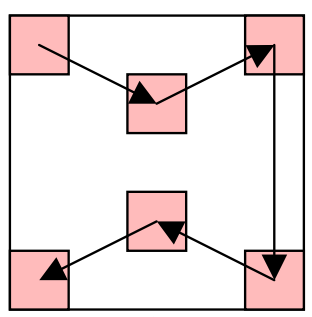

(b)
Figure 1 The schematic shows two possible arrangements of path points; adjacent (a) and non-adjacent path points (b). Both create different sonifications when applied to the same dataset.

\subsection{Span}

The span defines a neighborhood, which is associated with every path point. In principle, the path points could map directly into single entities of the data, however the span allows more innovative sonifications to be generated. For instance, we are able to specify irregularly shaped neighborhoods that can include spaces, such as depicted in Figure 2, this specific instance is a sonfication mask.

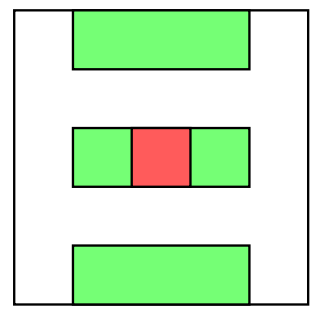

Figure 2 An irregular shaped span, which would be impossible to create by increasing the area a path point maps to.

\subsection{Span angle}

In addition to the ability to specify the span, we can also specify the angle of interception between the span and the path (values range from $0^{\circ}-180^{\circ}$ ).

Currently, all systems detailed in the literature, use a span that is perpendicular to the path (Figure 3a). However, angular spans are useful (Figure 3b). For example, there may be artifacts in the data that are aligned or grouped together at an angle, this may not perceived by a vertical (traditional) span but may become obvious when the span angle matches the angle of the data entities. Moreover, the span angle would dynamically change to keep particular alignments of the span, even when the orientation of the path varies.

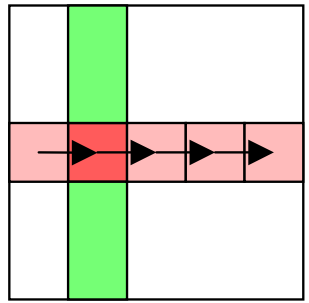

(a)

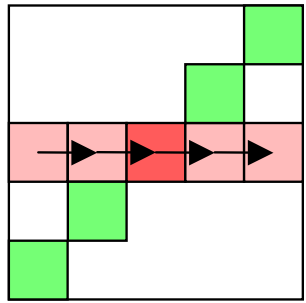

(b)
Figure 3 (a) Depicts a path that is using a span angle of $90^{\circ}$ (b) Shows a path that is using a span angle of $45^{\circ}$.

\subsection{Span envelope}

The span envelope designates the size of the span, dependent on the time and the position of the path. In most situations a fixed span envelope will be used (e.g. determined by a constant, or the height of the image). However, it is useful to dynamically change the span envelope (this may be imagined as two additional paths that surround the main path, Figure 4).

It is envisaged that the span envelope can be generated either manually or automatically by the system, which could optimize the overall length of the path, by using large spans (where there is little or no relevant information) and a smaller span (with extra path points) in locations of densely packed data points. 


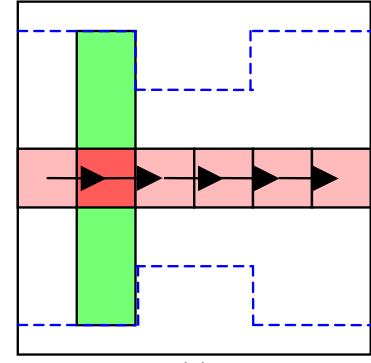

(a)

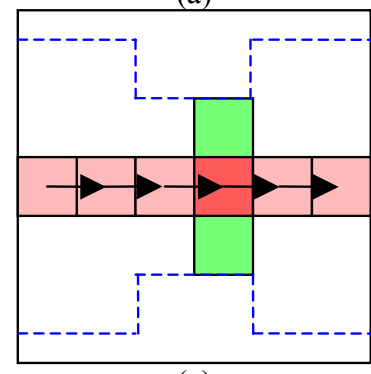

(c)

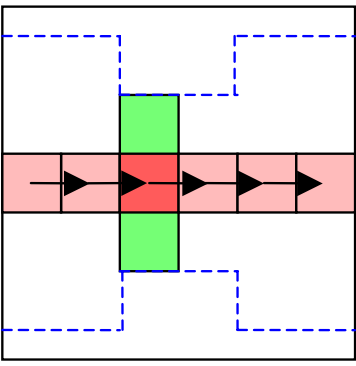

(b)

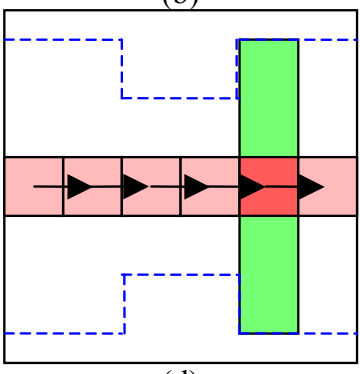

(d)
Figure $4 \mathrm{~A}$ sequence of images that show how the span size is changed as time progresses along the path. The span size depends on the span envelope (shown by the dotted line).

\subsection{Sonifying the path}

There are two stages to generate a sonification from a path. The first stage is to map the path onto the actual data set. This requires specifying how each span section maps onto the underlying data. In the situation when the resolution of the data and those of the path points are different, then registration of one to the other will need to take place, i.e. scaling, sampling or averaging. In our system, we have scaled the path and the sampled the data to register the span and path to the data.

Additionally, the mapping stage also depends upon assigning timing information. For example, time could continue linearly, or could speed up or slow down at various stages through the path. Time is treated as an additional variable on the path.

The second stage is to generate the sounds associated with each path point. This is dependant on (a) the data values that are currently under the span, and (b) the transfer function (describing how particular data values should be converted into sound).

\subsection{Example Paths}

To assist with the understanding of our model, we will describe how various paths can be used and developed in our model. Some of the paths are variations of those found in current literature, while others we newly present. Each path has advantages, disadvantages and particular challenges for perception; however, it is not our goal in this paper to discuss such perceptual issues, rather it is to demonstrate how the model can be applied to generate a wide range of sonification strategies.

\subsubsection{Scan line path}

The scan line path is the equivalent of the sweeping line method (as described in the introduction). It uses a path that crosses the data area in a straight line, a span angle of $90^{\circ}$, with a fixed span envelope. The height of the span envelope is equal to the height of the data (see Figure 5). As the span moves along the path all information that lies beneath the span and is simultaneously sonified.

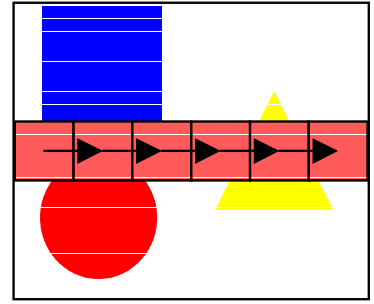

(a)

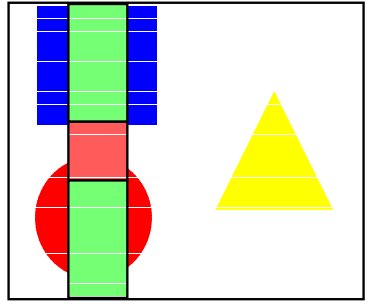

(b)
Figure 5 Scan line path. (a) Shows the position of the path points used in the scan line path (b) Indicates how the span is related to the each path point.

\subsubsection{Raster scan path}

This path demonstrates a method in which users would be able to understand the spatial relationship between different objects contained within the data set. The raster scan path uses a series multiple scans, which are parallel and equally spaced (see Figure 6a). A constant envelope is used such that the bottom of the envelope on one scan meets the top of the envelope on the next scan (see Figure 6b).

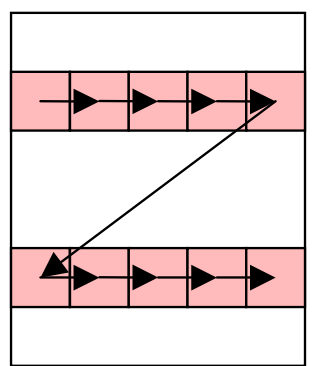

(a)

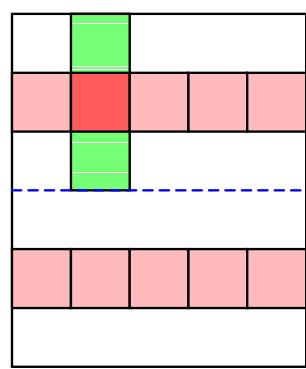

(b)
Figure 6 Raster Scan path. (a) Shows the path points and their order used in a raster scan path (b) Shows the span envelope for the path in (a).

\subsubsection{Zigzag path}

Unlike the raster scan path, the zigzag path uses a more continuous path to arrange the data into an order where every path point is adjacent to both the previous and the following point (see Figure 7). We believe that 
this might lower the mental demand placed on the users since the movement is not jumping from one edge of the data set to the other. But, depending on the span angle, some points may be sounded multiple times.

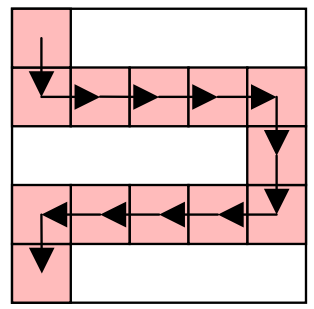

Figure 7 An example of a zigzag path.

\subsubsection{Spiral path}

This path traverses the data set in a spiral motion either from the outer edge (Figure $8 \mathrm{a}$ ) or from a center point (Figure 8b). Spiral paths have a particular challenge of what to do when the spiral path exceeds the physical boundary of the data. For example, if the data set is not square and an outward spiral motion is applied from the center point, then the path reaches the bottom boundary before all the information has been presented. There are three possible solutions, (1) ignore the remaining information, (2) jump to the next path point that is within the data set area, or (3) add some indication to the listener that the area being sonified is outside the boundary of the information space. Each of these approaches has their own implications to the users, which need to be studied before we can select the most appropriate option.

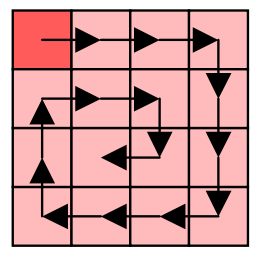

(a)

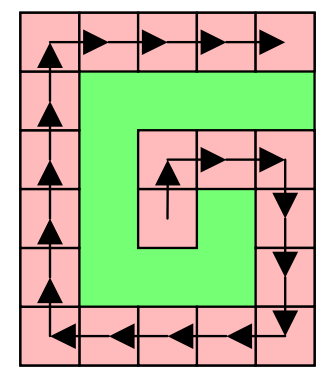

(b)
Figure 8 (a) and (b) show the two possible arrangements to create a spiral path sonification.

\subsubsection{Water droplet path}

This path aims to provide a way to convey information that is ordered by distance from a particular area. This is achieved by creating a circular span and increasing its diameter as the span moves along the path, shown in Figure 9. The path may be a fixed length, or extend to the edge of the dataset.

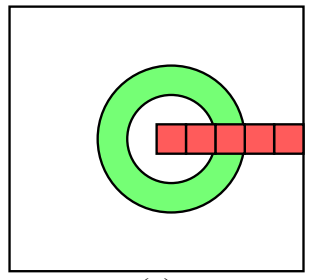

(a)

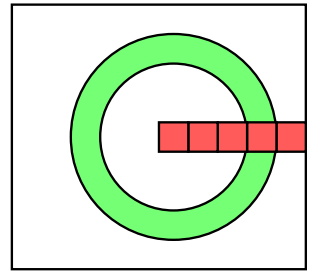

(b)
Figure 9 a) Shows the size of the span using the water droplet path at the being b) Shows how the span has increase in diameter as it has moved along the path.

\section{Prototype system}

We are currently developing a prototype system that demonstrates the model, Figure 10. If the system is being used by a blind user then a developer would be required to create a path or set of paths (which are independent of any image source) and that the blind users could load and use to sonify the image. In practice, we do not make a distinction between a developer and user and so any user can modify pre-existing tours.

The developer proceeds by creating a path, which represents particular areas of interest or key features within the data. Each path point is placed on a selectable grid, shown on the top left of Figure 10, the system draws a line connecting the previously placed path point to the newly introduced point.

When all the path points have been placed, the developer then needs to create the span. Again this is achieved by using a separate selectable grid in which the user indicates the relationship between the path point and the span area, shown on the right hand side of Figure 10. Currently, the system only implements the path and the span features of our model. However, in future versions, the developer would also setup the desired span angle and the span envelope.

Before the system can convert the abstract path into a tour based sonification, the system requires additional information about the conversion process. In particular, the system needs to know the area of the image that is to be sonified and how to fill increase the size of the abstract path so that it fills the specified area. Currently our system provides two sets of slider controls which both the developer and the user can adjust as necessary.

The first set of sliders control the 'target sonification area' that is to be sonified. The user specifies $\mathrm{x}$ and $\mathrm{y}$ coordinates for the top left hand corner, together with its width and height. The second set of sliders control the number of times the abstract path should be repeated within the 'target sonification area'. This process is often known as tiling. If the required amount of tiled repeats does not exactly fit the area, then the whole abstract path is scaled to fit.

Users can load pre-generated paths, along with any image, and listen to the sonification based on the path, span and other mapping controls. 


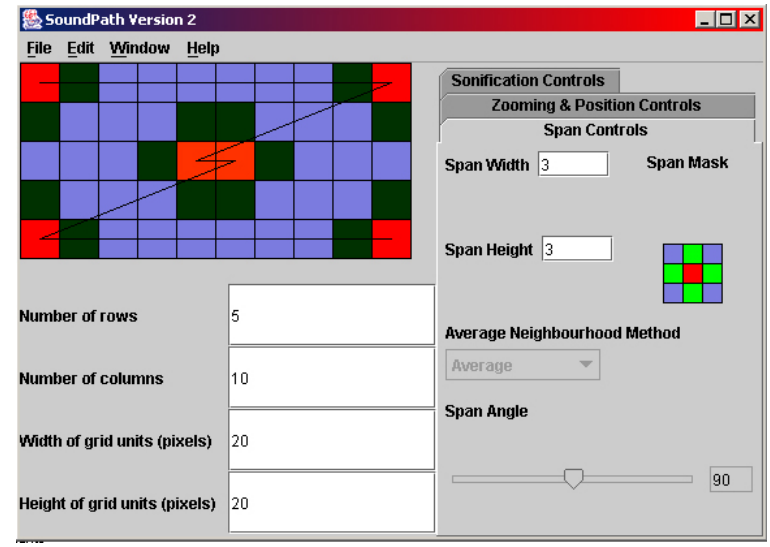

Figure 10 Our prototype system, which demonstrates the model.

\section{$5 \quad$ Results and discussion}

Currently, we are investigating the usability and effectiveness of new sonification paths in the representation of different information from equivalent mathematical visualization to image based sonification. In addition to studying what makes a usable/effective path, we are also considering if there is a relationship between different paths and the type of tasks that it is most effective in convey information for.

Initial results from the preliminary testing indicate that the raster scan allows users to correctly perceive spatial relationship between different data objects.

In conclusion, we have presented a model that unifies current tour based sonification methods by defining a set of terms which we believe will facilitate the development and expansion of sonifications. Our model allows researchers, developers and end users to convey the concepts of each path and what information will be presented, so that the most appropriate path can be selected when generating a sonification for a particular data set or task.

\section{Future work}

We plan to expand this research by continuing testing and developing new paths and the system. Currently we are carrying out an extended pilot study into a set of new paths that we have designed to eliminate paths that are completely unusable. After which, we plan to carry out an empirical study, which will look at the usability, effectiveness and the mental load that each path places on the users in relation to typical tasks that user might wish to carry out on various data sets.

One future plan is to investigate the possibility of automatically generating paths. The system will need to analyze what is 'interesting' in the data and create a tour that travels to the interesting features of the data [14], perhaps moves to an interesting area, sonify the information in detail and then move onto the next 'interesting' area.
Finally, it is important to investigate perceptual appropriateness of various tours because some paths may be more appropriate for a given task. For instance, the water droplet is useful to investigate a small area in detail (at the center) and the surrounding areas in less detail (as the span increases). Such perceptual evaluation is important.

\section{Acknowledgements}

We would like to thank all the people that participated in the pilot study of our system.

\section{References}

[1] D.L.Mansur, M.M.Blattner and K.I.Joy "Sound-Graphs: a numerical data analysis method for the blind". Journal of Medical Systems, 9(3), 1985, pp.163-174

[2] D.J.Bennett "Effects of Navigation and Position on Task when Presenting Diagrams to Blind People using Sound". Proc Diagrams 2002, Mark Hegarty, et al (eds), LNAI 2317, Springer

[3] M.H.Browns and J.Hershberger. "Color and Sound in Algorithm Animation”,IEEE Computer, 25(2):62-63, 1992

[4] S.Barrass and B.Zehner. "Responsive Sonification of Well-logs" Proceedings of International Conference on Auditory Displays (ICAD) 2000, Atlanta, April 2000

[5] R.S.Tannen. "Breaking the Sound Barrier: Designing Auditory Displays for Globla Usability". Proc of Human Factors \& the Web, Basking Ridge, USA, June 5, 1998

[6] F.James. "AHA: Audio HTML Access". Computer Networks and ISDN Systems, Volume 29, Issues 8-13, September, 1997, pp 1395-1404

[7] M.Blattner, D.Sumikawa and R.Greenberg. "Earcons and Icons: Their structure and common design principles", Human Computer Interaction, 4(1):11-44, 1989

[8] S.Helle, G.Leplâtre, J.Marila and P.Laine. "Menu Sonification in a Mobile Phone - a prototype study". Proc International Conference on Auditory Display, Espoo, Finland, July 29-August 1, 2001, pp 255-260

[9] A.R.Kennel. "AudioGraf: A diagram reader for blind people". Proceedings of ASSETS96, Vancouver, Canada, 1996, pp 51-56

[10] W.Yu and S.Brewster. "Comparing Two Haptic Interfaces for Multimodal Graph Rendering". In Symposium on Haptic Interfaces for Virtual Environment and Teleoperator Systems. 2002. Florida

[11] K.M.Franklin, J.C.Roberts. "Pie Chart Sonification". Proceedings of Information Visualization (IV03), London, UK, July, 2003, pp 4-9. IEEE Computer Press

[12] T.L.Bonebright, M.A.Ness, T.T.Connerley and G.R.MaCain. "Testing the Effectiveness of Sonified Graphs for Education: A Programmatic Research Project". Proc Int Conf on Auditory Displays, Espon, Finland, July 29-August 1, 2001

[13] W.D.Jones. "Sight for sore ears". IEEE Spectrum, February, 2004, pp 11-12

[14] Colton, S., Bundy, A. and Walsh, T. On the Notion of Interestingness in Automated Mathematical Discovery. International Journal of Human Computer Studies, Vol 53. No. 3, 351-375. 2000. 